\title{
Solving the Diagnostic Challenge: A Patient-Centered Approach
}

\author{
Norbert Donner-Banzhoff, MD, \\ MHSc \\ Department of General Practice, University \\ of Marburg, Marburg, Germany
}

\begin{abstract}
Arriving at an agreed-on and valid explanation for a clinical problem is important to patients as well as to clinicians. Current theories of how clinicians arrive at diagnoses, such as the threshold approach and the hypothetico-deductive model, do not accurately describe the diagnostic process in general practice. The problem space in general practice is so large and the prior probability of each disease being present is so small that it is not realistic to limit the diagnostic process to testing specific diagnoses on the clinician's list of possibilities. Here, new evidence is discussed about how patients and clinicians collaborate in specific ways, in particular, via a process that can be termed inductive foraging, which may lead to information that triggers a diagnostic routine. Navigating the diagnostic challenge and using patient-centered consulting are not separate tasks but rather synergistic.
\end{abstract}

Ann Fam Med 2018;16:353-358. https://doi.org/10.1370/afm.2264.

\section{INTRODUCTION}

T the French movie Irreplaceable (Médecin de Campagne, directed by Thomas Lilti), the old country doctor has to give up his practice for health reasons. He introduces his patients to a young colleague who is to replace him. A middle-aged patient seeks care for recent-onset headaches. The young doctor immediately takes over with direct questions, trying to establish location, severity, and associated features; however, no clear picture emerges from this very pointed questioning. The old doctor had noticed that the patient wanted to say something at the beginning of the consultation but was cut short by his young colleague. Once encouraged, the patient explains that the headache began when he started his new medication for diabetes.

How physicians arrive at their diagnoses has been the subject of many debates but of much less empirical research. The cognitive challenge is enormous, especially in generalist settings such as primary care. Previous theories do not adequately explain how clinicians cope, however. Here, I suggest a new approach based on recent evidence on primary care decision making.

I will first review previous theories of the diagnostic process and discuss their fit with the ecology of primary care. Drawing on evidence that has recently emerged, I will present a different conceptualization of the generalist diagnostic process.

\section{THE THRESHOLD APPROACH TO CLINICAL DECISION MAKING}

In 1980, Pauker and Kassirer ${ }^{1}$ proposed their threshold model of diagnosis. This model posits that when a physician is considering a specific disease, the action taken depends on 2 thresholds: a therapeutic (testing and treatment) threshold and a diagnostic (testing) threshold (Figure 1). Once the probability of disease rises above the therapeutic threshold, the physician stops the diagnostic process and takes action. This action may be therapy, but, depending on the context, could be other measures, such as referral. 


\section{Figure 1. The threshold model of the diagnostic} process.

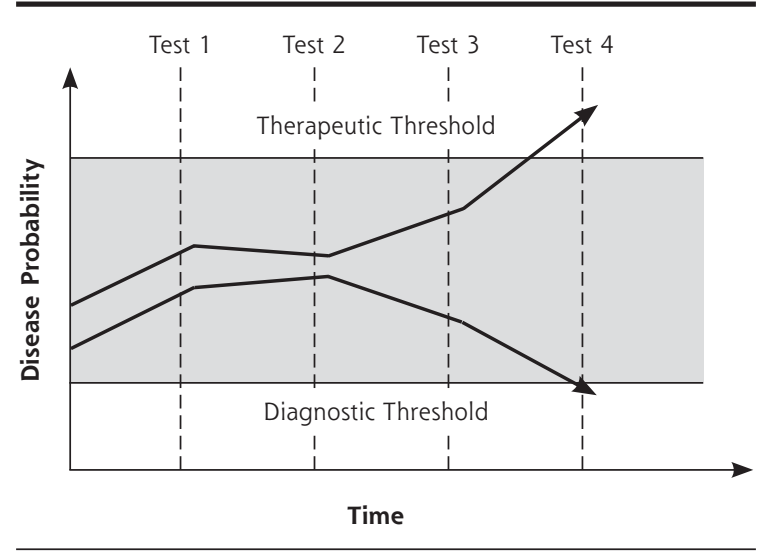

Note: The figure depicts 2 examples of diagnostic decision making. The shaded area represents the state of diagnostic uncertainty. The top example shows eventual crossing of the upper boundary (therapeutic threshold), indicating ruling in of disease and start of treatment or other intervention. The bottom example shows eventual crossing of the lower boundary (diagnostic threshold), indicating ruling out of disease.

On the other hand, if the disease probability falls below the diagnostic threshold, the physician considers the disease to be absent and stops collecting data relevant for confirming or rejecting that diagnosis. As long as the disease probability lies between the diagnostic and therapeutic thresholds, further diagnostic testing is justified until the case is solved, either by crossing the therapeutic threshold upward (disease assumed to be present) or crossing the diagnostic threshold downward (disease assumed to be absent).

Factors influencing the probability levels at which the diagnostic and therapeutic thresholds are set include disease severity and the benefits and harms of tests and treatments. Other formal models to calculate thresholds have been proposed..$^{2-4}$ They have descriptive as well as normative values. The latter apply when the threshold model helps clinicians align their diagnostic practice with their own values or, better but harder, those of their patients.

Although the threshold model has an intuitive appeal, recent research from primary care shows that the explicit testing of specific hypotheses (deductive testing) may occur in less than $40 \%$ of diagnostic episodes. ${ }^{5}$ Strategies not directed at specific disease hypotheses are more common and capture more diagnostic cues than hypothesis testing. ${ }^{5}$ Not all clinical encounters end with disease probability crossing 1 of the 2 thresholds and resulting in decision. Once acute serious disease has been excluded, clinicians use expectant strategies (watchful waiting). ${ }^{6}$ Lastly, the threshold model is focused on the physician; the patient is a completely passive party in this view. This view contradicts empirical research showing that patients take an active part in clinical (diagnostic) decision making. ${ }^{7.8}$

\section{THE ECOLOGY OF THE CLINICAL PROBLEM SPACE}

The threshold approach assumes that the clinical problem space is clearly bounded and mostly filled with identifiable specific diseases. This is not true in primary care, however. Here, the problem space is almost infinite and largely undifferentiated, and the prevalence of serious and specific diseases is low. ${ }^{9}$ For example, even among patients presenting with chest pain, only $1.5 \%$ to $3.5 \%$ have an acute coronary syndrome. ${ }^{10} \mathrm{Sim}$ ilarly, merely $1 \%$ of cases of abdominal pain are caused by neoplastic disease. ${ }^{11}$ Pulmonary embolism, dissecting aortic aneurysm, and many other life-threatening conditions are too rare to be even quantifiable at the primary care level. ${ }^{12}$ These probabilities are below any reasonable diagnostic threshold. In other words, if we take the threshold model seriously, we would consider most serious diseases to be excluded at the beginning of the consultation!

A common tenet of evidence-based medicine is that to rule out disease, sensitive tests are preferred (captured by the shorthand term sn-out). ${ }^{13}$ In low-prevalence settings, however, the likelihood of disease being present after testing, that is, the negative predictive value for disease, is invariably small. Even highly sensitive tests do not usefully modify this low probability. For instance, in a patient presenting with chest pain in primary care, the prevalence of acute coronary syndrome is approximately $2.5 \% .{ }^{11}$ If the patient is fairly young (younger than 65 years if female or younger than 55 years if male) and not feeling chest pressure or tightness, the likelihood drops to $0.26 \%$. But with positive findings, such as known coronary heart disease or a request for an urgent home visit, it rises to a clinically relevant $42 \%$, crossing the diagnostic threshold upward. ${ }^{14}$ In other words, with low prevalence, sensitive tests often are seldom informative. ${ }^{15}$

This situation conflicts with the threshold model's implicit assumption that the probability of disease is above the diagnostic threshold and below the therapeutic threshold at the beginning of the diagnostic process. How do primary care physicians arrive at disease probabilities above the diagnostic threshold in the first place?

The challenge is even greater because the physician has to rule out a large number of potentially serious conditions. Moreover, most presentations in primary care are ambiguous, and several different explanations are possible even if only remotely. Finally, many clinically important health issues cannot be captured by conventional disease categories. 


\section{SEARCHING SPACES}

What physicians actually do, especially early in the patient encounter, thus requires new descriptions. I propose that physicians search this expanded problem space first and that the patient has the leading role here.

\section{Inductive Foraging and Triggered Routines}

An analysis of 282 primary care consultations and 163 diagnostic episodes has identified a process called inductive foraging that has been shown to precede the formulation of specific hypotheses. ${ }^{5,16}$ This process is the initial invitation to the patient to describe his or her problem. Usually, it goes far beyond the patient stating what is subsequently recorded as the presenting complaint. Patients spontaneously mention further symptoms, functional associations, and often also their own explanations or concerns. If they are allowed to do so without interference, they will lead their clinician to symptoms and problems as they perceive them, and will thus provide an exploration of the problem space.

To give some examples, a 63-year-old man with tiredness and depressed mood mentions to me his recent difficulties buttoning his shirt, thus providing a hint for early Parkinson disease. A 67-year-old retired plumber reports frequent episodes of cough in the recent past. Pondering whether to order spirometric testing, I nearly miss him mentioning that he regularly plays the tuba in the local brass band, which reassures me about his lung function.

Against the background of an almost infinite problem space, an exploration by the physician asking direct and mostly closed-ended questions is hardly realistic in generalist settings. Once the patient has been interrupted, he or she usually switches into a passive mode and answers only those questions related to problems the physician can think of. It is obvious that important and unexpected points can be missed after this kind of premature closure. This outcome is clearly shown by the introductory example about drug-induced headache. It is unlikely that the young physician would have arrived at the hypothesis of a medication adverse effect herself, or she may have arrived there only after lengthy questioning and cognitive effort. Giving the patient sufficient time for his initial description and encouraging him by active listening are thus not only a matter of kindness, but also improve the diagnostic yield and efficiency of the consultation.

After the patient has helped define the problem space, physicians explore limited areas by direct questions but without guidance by specific hypotheses, a process called a triggered routine (Figure 2). For instance, a patient reporting vomiting is asked about abdominal pain and bowel movements. The young physician questioning her patient about the characteristics of his headache is another example. Inductive foraging and triggered routines do not require defined hypotheses. Testing hypotheses too early can even be harmful because important information might be missed. As our previous research has shown, ${ }^{5}$ these exploratory strategies provide sufficient information so that evaluation of specific diagnostic hypotheses is needed in less than one-half of consultations. Only for this remaining minority do primary care physicians need to gather additional data guided by specific diagnostic hypotheses as suggested by the hypothetico-deductive model emerging from a seminal study by Elstein et al. ${ }^{17}$

\section{The Hypothetico-Deductive Model of Diagnosis}

The hypothetico-deductive model has been the predominant theory of diagnostic reasoning in medicine. ${ }^{17}$ According to this model, early in the encounter with the patient, possible explanations (hypotheses) come to the physician's mind. These hypotheses guide further data collection aiming at either confirmation or refutation. This model, revolutionary at the time it was introduced, was based on observation of hospital physicians reflecting on their reasoning (thinking aloud) while evaluating prepared, standardized patients. ${ }^{17}$ But this setting is likely to suggest specific hypotheses more often than would real-world primary care patients, whose symptoms often cannot satisfactorily be explained within a biomedical framework. ${ }^{18}$

\section{Confirmation Bias or Rational Falsification Strategy?}

In the literature on faulty clinical reasoning, confirmation bias is frequently mentioned as a source of diagnostic error. ${ }^{19,20}$ Physicians influenced by this bias would search only for information confirming their hypotheses, neglecting contradicting findings. Once a large problem space has to be searched, however, what is usually criticized becomes a rational strategy, that is, giving attention to confirming evidence.

\section{Figure 2. Problem space of the diagnostic task.}

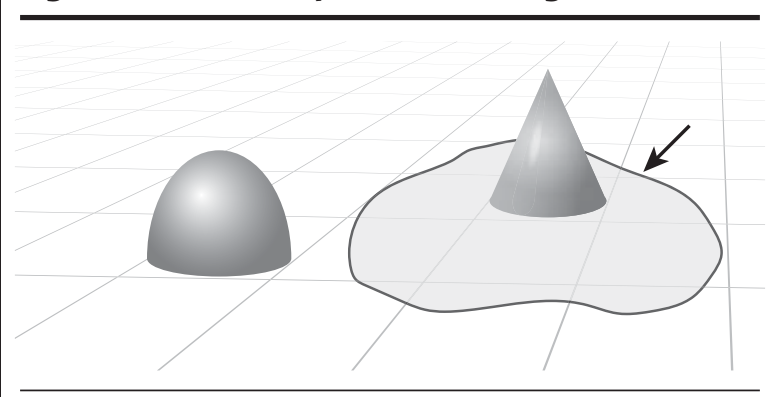

Note: A problem space in which the outstanding geometric forms denote unusual and relevant findings (symptoms), typically identified by inductive foraging with patient input. Arrow points to a shaded limited problem area to be explored by a triggered routine. 
As suggested above, primary care diagnosis starts with the assumption that serious conditions can be ruled out. During the consultation, this assumption is being put to a critical test by searching for findings suggesting a specific disease that, if present, leads to further inquiry. In other words, clinicians clearly use a falsification strategy by exploring the problem space in the way described above. ${ }^{16}$ At this early stage, they do not invest in ascertaining negative findings, because these carry little information. As long as disease prevalence is low, physicians are therefore perfectly justified in searching for suggestive (positive) findings. ${ }^{21,22}$ Only after the likelihood of a particular diagnosis has been raised by findings pointing in that direction does the absence of findings becomes informative.

In this process, clinicians make use of the fact that specific diseases as well as pathologic findings (symptoms, signs, test abnormalities, etc) occur much less frequently than in $50 \%$ of cases. ${ }^{23}$ Although the absence of disease is the default hypothesis, clinicians search the problem space for findings suggesting a specific disease. Obviously, criteria with high specificity are particularly helpful at this stage: if they are met, they indicate the presence of disease with high probability. Their presence does not necessarily mean that they are specific for any particular disease. Primary care physicians group diseases together to make their large number manageable (eg, "nasty esoteric viruses"). ${ }^{24}$ Findings are useful when their presence indicates an area worthy of further investigation. A patient with respiratory infection mentioning the symptom of dyspnea would trigger a new line of inquiry, as opposed to acceptance of the first impression of benign and self-limiting disease; this narrower problem space might include pneumonia, obstructive lung disease, or congestive heart failure. The concept of "red flags" comes close to this idea of searching problem spaces without necessarily specifying any particular hypothesis. Idiosyncratic impressions that something does not fit or does not feel right can be similarly helpful. ${ }^{25,26}$

\section{ADAPTIVE STRATEGIES NEEDING PATIENT INVOLVEMENT}

Relevant disease cannot satisfactorily be ruled out by absence of any particular sign or even several signs, but only by a thorough inductive and collaborative search of the problem space (Figure 2). Its cornerstone is a confident patient having sufficient time and encouragement to mention all things that are different, worrisome, or both. Clinicians working in intimidating settings or interrupting their patients' too soon during the inductive foraging phase are at a disadvantage. As the introductory example of drug-induced headache shows, such physicians will have to think of every possible explanation themselves and seek out related data, thereby potentially missing important findings or explanations. The accuracy of the diagnostic process is thus highly dependent on the quality of the clinicianpatient relationship. The approach described here for initial history taking and examination may be extended into shared decision making about tests such as imaging or invasive procedures. ${ }^{27}$

Human beings adapt their cognitive strategies to the environment and to the task at hand ${ }^{28}$ The physicians taking part in the seminal study of Elstein et a ${ }^{17}$ must have assumed that the case stories portrayed by actors or described on paper had a defined solution. In real life, clinicians face the alternate challenge of a potentially infinite problem space (described above) and patients with diffuse, ambiguous findings often defying any medical explanation. ${ }^{16}$ They turn to hypothetico-deductive testing only if the problem space has been narrowed sufficiently and relevant information is still missing.

The phenomenology described above does not exclude other processes relevant for medical diagnosis, such as feelings of alarm or reassurance, ${ }_{1}^{25}$ rules of thumb, ${ }_{r}^{29}$ or pattern recognition. ${ }^{30,31}$ The last, which is certainly common and relevant, works well when the clinician is aware of the full range of pertinent symptoms and signs. Inductive foraging can prevent premature arrival at unjustified conclusions. ${ }^{16}$

Patient and physician collaboratively searching the problem space best describes the phenomenology of the primary care diagnostic process. (Figure 3 shows related tactics and potential pitfalls.) This model of collaborative searching accommodates criticisms brought forward against previously formulated 


\section{Table 1. Key Messages on the Diagnostic Process in Primary Care}

- In generalist settings, the problem space of possible problems (diagnoses) is almost infinite.

- Recent research suggests that established descriptions and models do not adequately capture what clinicians do to arrive at a diagnosis.

- Searching the problem space by inductive foraging and triggered routines is emerging as a diagnostic strategy that can be adapted to the generalist setting.

- The patient has a leading role in this collaborative process.

theories, especially the threshold and hypotheticodeductive models. Although relevant data have mostly been derived from primary care, I believe that this model may apply to any clinical setting where more than 1 disease is of interest.

\section{CONCLUSION: PATIENT-CENTEREDNESS PAYS!}

Patient-centeredness has been persuasively argued to be an essential feature of good family practice, most notably by McWhinney and Stewart and their colleagues. ${ }^{32,33}$ Yet patient-centeredness is far from generally adopted, and failure to listen is one of the most common criticisms of clinician behavior. ${ }^{34-36}$ Building an effective relationship and making a diagnosis are often seen as separate skills, but I would like to stress that these tasks are synergistic. Diagnostic efficiency is very hard to achieve without the patient's contribution. Every diagnostic inquiry is a new collaborative adaptation within the clinical problem space. Self-interest and ultimate time saving should lead all primary care generalists to involve their patients in early shared inquiry, which I and colleagues have called inductive foraging. This exploration of how clinicians arrive at diagnoses can be summarized in several key messages that can inform primary care practice (Table 1 ).

To read or post commentaries in response to this article, see it online at http://www.AnnFamMed.org/content/16/4/353.

Key words: clinical decision making; diagnosis; hypothetico-deductive reasoning; inductive foraging; physicians, primary care; primary health care; threshold model

Submitted October 6, 2017; submitted, revised, March 15, 2018; accepted April 12, 2018.

Acknowledgments: I thank Stefan Bösner, University of Marburg, Germany; George Freeman, Imperial College, London, UK; Wolfgang Gaissmaier, University of Konstanz, Germany; Alison Hill, retired general practitioner, Pangbourne, UK; and Thomas Kühlein, University Medicine Erlangen, Germany, for their thoughtful comments on previous versions of this article.

\section{References}

1. Pauker SG, Kassirer JP. The threshold approach to clinical decision making. N Engl J Med. 1980;302(20):1109-1117.

2. Tsalatsanis A, Hozo I, Vickers A, Djulbegovic B. A regret theory approach to decision curve analysis: a novel method for eliciting decision makers' preferences and decision-making. BMC Med Inform Decis Mak. 2010;10:51.

3. Vickers AJ, Elkin EB. Decision curve analysis: a novel method for evaluating prediction models. Med Decis Making. 2006;26(6):565-574.

4. Sox HC. Medical Decision Making. Philadelphia, PA: American College of Physicians; 2007.

5. Donner-Banzhoff N, Seidel J, Sikeler AM, et al. The phenomenology of the diagnostic process: a primary care-based survey. Med Decis Making. 2017;37(1):27-34.

6. Irving G, Holden J. The time-efficiency principle: time as the key diagnostic strategy in primary care. Fam Pract. 2013;30(4):386-389.

7. Gill VT, Pomerantz A, Denvir P. Pre-emptive resistance: patients' participation in diagnostic sense-making activities. Sociol Health IIIn. 2010;32(1):1-20.

8. Epstein RM, Franks P, Shields CG, et al. Patient-centered communication and diagnostic testing. Ann Fam Med. 2005;3(5):415-421.

9. Green LA, Fryer GE Jr, Yawn BP, Lanier D, Dovey SM. The ecology of medical care revisited. N Engl J Med. 2001;344(26):2021-2025.

10. Haasenritter J, Biroga T, Keunecke C, et al. Causes of chest pain in primary care-a systematic review and meta-analysis. Croat Med J. 2015;56(5):422-430.

11. Viniol A, Keunecke C, Biroga T, et al. Studies of the symptom abdominal pain-a systematic review and meta-analysis. Fam Pract. 2014;31(5):517-529.

12. Bösner S, Becker A, Haasenritter J, et al. Chest pain in primary care: epidemiology and pre-work-up probabilities. Eur J Gen Pract. 2009; 15(3):141-146.

13. Guyatt G, Sackett DL, Haynes RB. Evaluating diagnostic tests. In: Haynes RB, Sackett DL, Guyatt GH, Tugwell P, eds. Clinical Epidemiology: How to do Clinical Practice Research. 3rd ed. Philadelphia, PA: Lippincott Williams \& Wilkins; 2005:273-322.

14. Haasenritter J, Bösner $S$, Vaucher $P$, et al. Ruling out coronary heart disease in primary care: external validation of a clinical prediction rule. Br J Gen Pract. 2012;62(599):e415-e421.

15. Connell FA, Koepsell TD. Measures of gain in certainty from a diagnostic test. Am J Epidemiol. 1985;121(5):744-753.

16. Donner-Banzhoff N, Hertwig R. Inductive foraging: improving the diagnostic yield of primary care consultations. Eur J Gen Pract. 2014; 20(1):69-73.

17. Elstein AS, Schulman LS, Sprafka SA. Medical Problem-Solving: An Analysis of Clinical Reasoning. Cambridge, MA: Harvard University Press; 1978.

18. Jones R, Barraclough K, Dowrick C. When no diagnostic label is applied. BMJ. 2010;340:c2683.

19. Wolf FM, Gruppen LD, Billi JE. Differential-diagnosis and the competing-hypotheses heuristic. A practical approach to judgment under uncertainty and Bayesian probability. JAMA. 1985;253(19): 2858-2862.

20. Dawson NV. Physician judgment in clinical settings: methodological influences and cognitive performance. Clin Chem. 1993;39(7): 1468-1480.

21. Klayman J, Ha YW. Confirmation, disconfirmation, and information in hypothesis-testing. Psychol Rev. 1987;94(2):211-228.

22. Oaksford M, Chater N. Optimal data selection: revision, review, and reevaluation. Psychon Bull Rev. 2003;10(2):289-318.

23. McKenzie CRM, Chase VM. Why rare things are precious: how rarity benefits inference. In: Todd PM, Gigerenzer G, eds. Ecological Rationality: Intelligence in the World. Oxford, UK: Oxford University Press; 2012:310-334. 
24. Gill CJ, Sabin L, Schmid CH. Why clinicians are natural Bayesians. BMJ. 2005;330(7499):1080-1083.

25. Stolper E, van Royen P, Dinant GJ. The 'sense of alarm' ('gut feeling') in clinical practice. A survey among European general practitioners on recognition and expression. Eur J Gen Pract. 2010;16(2):72-74.

26. Granier S, Owen P, Pill R, Jacobson L. Recognising meningococcal disease in primary care: qualitative study of how general practitioners process clinical and contextual information. BMJ. 1998; 316(7127):276-279.

27. Berger ZD, Brito JP, Ospina NS, et al. Patient centered diagnosis: sharing diagnostic decisions with patients in clinical practice. BMJ. 2017;359:j4218.

28. Todd PM, Gigerenzer G, eds. Ecological Rationality: Intelligence in the World. Oxford, UK: Oxford University Press; 2012.

29. Andre M, Borgquist L, Foldevi M, Molstad S. Asking for 'rules of thumb': a way to discover tacit knowledge in general practice. Fam Pract. 2002;19(6):617-622.
30. Hobus PP, Schmidt HG, Boshuizen HP, Patel VL. Contextual factors in the activation of first diagnostic hypotheses: expert-novice differences. Med Educ. 1987;21(6):471-476.

31. Schmidt HG, Norman GR, Boshuizen HPA. A cognitive perspective on medical expertise - theory and implications. Acad Med. 1990; 65(10):611-621.

32. McWhinney IR. A Textbook of Family Medicine. 2nd ed. New York, NY: Oxford University Press; 1997.

33. Stewart M. Patient-Centered Medicine: Transforming the Clinical Method. Thousand Oaks, CA: Sage Publications; 1995.

34. Marvel MK, Epstein RM, Flowers K, Beckman HB. Soliciting the patient's agenda: have we improved? JAMA. 1999;281(3):283-287.

35. Rhoades DR, McFarland KF, Finch WH, Johnson AO. Speaking and interruptions during primary care office visits. Fam Med. 2001;33(7): 528-532.

36. Beckman HB, Frankel RM. The effect of physician behavior on the collection of data. Ann Intern Med. 1984;101(5):692-696.

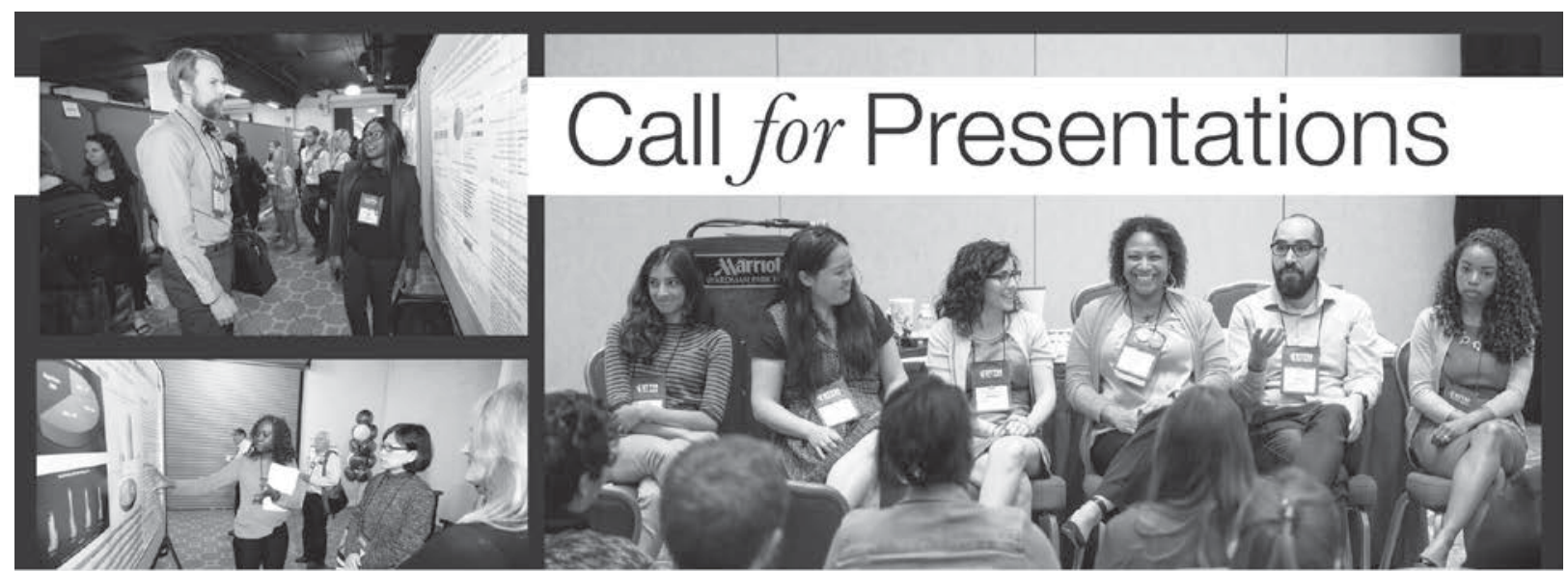

Submit today at www. stfm.org/annual · Submission Deadline: September 10, 2018

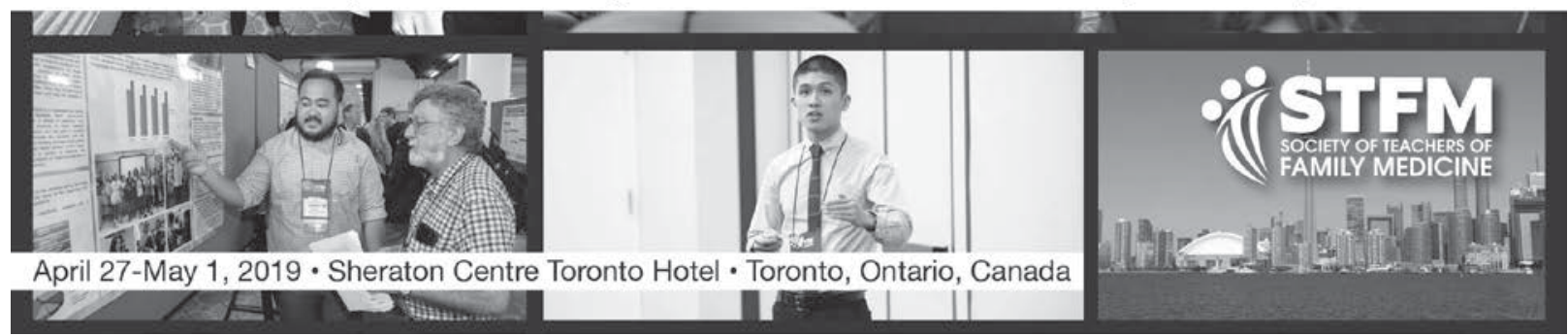

